\title{
Reforma y Políticas Juveniles En México: Avances y Retrocesos Institucionales ${ }^{1^{*}}$.
}

\section{Karla Valverde Viesca ${ }^{2}$}

\author{
Tipo de trabajo: Artículo
}

Material original autorizado para su primera publicación en el Journal de Ciencias Sociales de la Facultad de Ciencias Sociales de la Universidad de Palermo

\section{Recibido 24-8-2012}

\section{Aceptado 29-9-2012}

\section{Resumen:}

El proceso de reforma impulsado durante la década de los ochenta, en diversos países del mundo privilegió, como en el caso mexicano, la liberalización económica y la consolidación del ámbito político-electoral, aplazando la construcción de un andamiaje institucional para diseñar e instrumentar políticas, planes y programas asociados con el bienestar y el desarrollo de la sociedad. Si bien son muchos los problemas que aquejan a la sociedad mexicana, en particular nos interesa identificar las características de las políticas juveniles. Proyecciones socio-demográficas para el año 201 calculan que entre una población total aproximada de 112 millones de habitantes en México, 29 millones 519 mil personas, es decir, el 27\%, pertenecen al grupo etario de 15 a 29 años de edad. En este trabajo se enfatiza el tránsito entre políticas e instituciones centralizadas, coyunturales y sectoriales hacia aquellas que buscan el desarrollo local y la atención de la diversidad y complejidad que de ello deriva, particularmente en los ámbitos de educación, empleo y participación juvenil. Asimismo, sostiene que dichos avances en el diseño de las políticas juveniles en México ha tenido poco impacto debido, entre otras cosas, a la inercia institucional que debilita y fragmenta la construcción de un nuevo entramado.

Palabras Claves: Políticas públicas, juventud, diseño institucional, desarrollo

\footnotetext{
${ }^{1 *}$ En sus orígenes, este trabajo se elaboró en el marco del Proyecto PAPIIT IN 303806 Hacia un nuevo paradigma para el desarrollo, financiado por la Universidad Nacional Autónoma de México. Posteriormente, se insertó en el proyecto APYO-COMPL2009-118430-S Nuevas reglas para el desarrollo social en México, financiado por el CONACYT. Agradezco en la actualización de datos e información para esta investigación, el apoyo de los alumnos de la Licenciatura en Ciencias Políticas y Administración Pública de la Facultad de Ciencias Políticas y Sociales de la UNAM: Abril Herrera Chávez y Betzabé Mendoza Paz, becarias de los proyectos, así como a Luis Miguel Juárez Figueroa quien realizó su servicio social en el mismo y a José Antonio Hernández Sánchez, quien colaboró como becario en la integración final del documento. No quiero dejar de mencionar, que los comentarios siempre oportunos de Rocio Canudas, fueron fundamentales para la reflexión general del artículo.

2 Doctora en Ciencia Política. Profesora de Tiempo Completo en la Facultad de Ciencias Políticas y Sociales de la Universidad Autónoma de México. Actualmente preside el Consejo Mexicano de Investigación en Ciencia Política (COMICIP).

E-mail: viesca@unam.mx
} 


\section{REFORMS IN POLITICS FOR YOUTH IN MEXICO}

\section{Abstract:}

As in different countries of the world, in Mexican case the reform process promoted in eighties privilege economic liberalization and consolidation of electoral sphere delaying the construction of an institutional framework for the designing and implementing of plans and programs associated with welfare and social development. With these context, this paper focus in description of youth policies. Some socio demographic projections, establish that in 2015 Mexico will have a total population around 112 million and $27 \%$, this mean almost 29 million of people, will be localized in group between 15 and 29 years old. Emphasis is made in policies and institution transition from those who promote local development and care about particular complex areas such as employment and youth participation. Paper arguments that advances in the design of youth policies in Mexico, have fragile impact because the institutional inertia weaken and fragments the construction of a new structure to strengthen youth citizenship in order to encourage youth participation and turning them into subjects and actors of rights.

Keywords: Public policy, youth, institutional design, development

\section{Introducción}

Durante las últimas dos décadas, la construcción y cimentación de un andamiaje institucional para la vida democrática en México, ha avanzado notablemente. En efecto, la creación de una serie de instituciones autónomas y la ciudadanización de los procesos electorales cada vez hacen menos cuestionable el sistema democrático en el ámbito político-electoral. Sin embargo, el impulso a la "democratización" parece centrarse sólo en ese ámbito dejando de lado el diseño de instrumentos y mecanismos que incidan en lo que algunos señalan como democracia, también en los ámbitos económico y sociales ${ }^{3}$.

A diferencia de otros países de América Latina y Europa del Este, algunos autores como Ortega Riquelme (2006) explican como la economía y la política mexicanas han recorrido caminos distintos y no han logrado concordar. Además, el tránsito entre un modelo estatal intervencionista y aquél que privilegia la liberalización económica, entre un sistema político autoritario y uno que aloja la democracia se ha realizado amén de un costo social a todas luces profundo.

Si bien este contexto afecta a la población en general, es el grupo etario 4 localizado entre los 14 y los 29 años de edad el que parece más vulnerado. En este trabajo, se analiza la condición juvenil que define a México en los últimos años. En particular, se resalta el diseño de las políticas juveniles que en México ha tenido un débil impacto en la construcción de ciudadanía. El documento, está dividido en tres secciones. En la primera, se presenta un breve recorrido por el proceso de reforma estatal mexicano que definió la década de los ochentas. En un segundo apartado, se describen algunas condiciones socio-demográficas que definen a la población juvenil en México y se enfatizan

\footnotetext{
${ }^{3}$ Aunque este es un tema en debate, puede consultarse: Larry Diamond y Leonardo Morlino, The Quality of Democracy, Center on Democracy, Development, and The Rule of Law (CDDRL) Stanford Institute on International Studies, Working Paper No. 20, 21 de septiembre de 2004. De los mismos autores también el libro, Assesing the Quality of Democracy, John Hopkins University Press, 2005.

${ }^{4}$ Me refiero al término derivado del Latin aetas, edad, que se refiere a varias personas que tienen la misma edad y es aceptado en la literatura sobre el tema.
} 
algunos avances y retrocesos que en materia juvenil caracterizan a México, en el ámbito educativo, laboral y de participación política.

\section{Reformas político-institucionales, económicas y sociales}

En el caso mexicano, la transición que tiene como telón de fondo una reforma del Estado se definió a partir de dos ámbitos: el político y el económico. El primero, se caracterizó por un cambio en el sistema político tradicional basado en estructuras autoritarias como el presidencialismo, el partido único, el corporativismo y una clase política homogénea que dio paso a un presidencialismo acotado, un sistema de partidos competitivo, el ascenso de una nueva clase política al poder y la extinción del modelo corporativista. Por otra parte Faundez y Valverde (1998) señalan que el ámbito económico, dejó atrás el proteccionismo y los subsidios estatales para dar marcha a una liberalización de los mercados y a la competencia regional. Vale la pena señalar, mientras las dinámicas política y económicas siguieron su paso, parecieron dejar a un lado o al menos postergar, el ámbito social.

En términos muy sintéticos, es posible ubicar los orígenes de una reforma política en México durante los años setenta cuando se crea la primera Ley de Organizaciones Federales y Procesos Electorales (LOFPE) aunque en realidad se consolida a mediados de la década de los ochenta, con la creación del Código Federal Electoral (COFEL). Desde entonces y sumados los esfuerzos emprendidos con anterioridad $^{5}$, se avanza sistemáticamente en un proceso de reforma y ciudadanización, tanto para institucionalizar el proceso electoral, como para independizarlo del gobierno. En este sentido, destaca la creación en 1990 del Código Federal de Instituciones y Procedimientos Electorales (COFIPE) del cual surgen dos organismos fundamentales: el Instituto Federal Electoral (IFE) encargado de organizar todo el proceso electoral; y, el Tribunal Federal Electoral (TRIFE) que en 1996 cambia su nombre por el de Tribunal Electoral del Poder Judicial de la Federación (TEPJF y es el órgano encargado de calificar la elección.

En el ámbito económico, la reforma aunque inicia a principios de la década de los ochenta surge con mayor fuerza en 1989, sobre todo como consecuencia de los problemas que la crisis económica profundizó, de manera particular en los países latinoamericanos durante la llamada década perdida. Ya en los noventa, durante la administración del presidente Salinas de Gortari, se consolida como parte de una reforma estructural o del Estado. En sí, la reforma económica se presenta en México como una racionalización de los recursos que cumple con la premisa de un Estado mínimo al reorganizar su actividad y reestructurar sus funciones económicas. Pero, el "adelgazamiento del Estado" se presenta como una contradicción entre las viejas estructuras e instituciones sociales que, a la fecha, no se han transformado a la par de los requerimientos que la integración y regionalización imponen.

Otro eje de esta reforma se relaciona con el papel de la descentralización, por lo general, encaminado al fortalecimiento de los municipios y gobiernos federales, el establecimiento de coordinaciones fiscales, la canalización de recursos a los programas sociales. Sin embargo, la supuesta descentralización de funciones no se ha traducido aún en mayor autonomía política, administrativa o control de recursos en el ámbito estatal y municipal (Millán, 1994).

\footnotetext{
${ }^{5}$ Antecedentes significativos son: 1952, la creación de la Comisión Federal Electoral (CFE) y del Registro Nacional de Electores (RNE); Ley Electoral de 1973; Ley Federal de Organizaciones Políticas y Procesos Electorales (LFOPPE) en 1977; en 1987, surge el Código Federal Electoral (COFEL) y el Tribunal de lo Contencioso Electoral (TRICOEL).
} 
Con respecto a su dimensión social, la reforma estatal promovió una retirada de los "tutelajes" para promover una mayor autonomía e iniciativa de otros agentes de la sociedad. En ese sentido, el impulso al desarrollo se presenta como un elemento sustantivo para legitimar el proceso de reforma en sus diversos ámbitos. Frente al cúmulo de intereses políticos en las últimas décadas es posible identificar un dilema frente al cual la política social se convierte en un mecanismo eficiente para intercambiar legitimidad política, acceso a recursos y apertura de canales de participación, a cambio de una compensación social. Ello explica en parte, por qué la canalización de recursos hacia el rubro de gasto social no garantiza la obtención de resultados alentadores.

Si bien es cierto que esta contradicción evidencia que en las últimas dos décadas los avances en términos de una estructura institucional como sustento del desempeño económico y social en México permanezcan rezagados ${ }^{6}$, particularmente en contraste con los logros obtenidos en los ámbitos de la construcción de instituciones democráticas, la búsqueda de la estabilidad económica, el impulso a la apertura comercial, así como en el proceso de reestructuración de Estado y de sus funciones, es importante señalar que para contrarrestar esta tendencia, en el año 2005 se aprobó la Ley General de Desarrollo Social que, entre otras cosas, busca promover la participación social más allá de los mecanismos electorales, es decir, como un principio que a partir del marco jurídico “....no resuelve por sí mismo el problema estructural de la pobreza y la desigualdad, pero parece establecer una serie de directrices que guían el diseño y elaboración de políticas públicas, para coordinar y unificar la acción institucional y programática en lo que a políticas sociales y desarrollo se refiere (Valverde y Palma, 2006, p. 26.).

Pese a este esfuerzo, nos encontramos con que en México el marco institucional se separa cada día más de la realidad que lo rodea, logrando acciones parciales, focalizadas y discrecionales, solo cuando son necesarias. Las instituciones relacionadas con el desarrollo, el bienestar y las políticas sociales reflejan un contenido que dio respuesta a relaciones añejas entre los diversos actores de la sociedad, sobre todo si entendemos a la política social como un conjunto de estrategias y acciones que coordinadas institucionalmente se dirigen a la población en general para garantizar su acceso a un nivel básico de vida que les permita ejercer plenamente sus derechos ciudadanos.

Así, el estudio sobre los avances y retrocesos institucionales para el caso mexicano se inscribe necesariamente en un proceso de modernización que tiene como sustento reformas políticas y económicas que buscan adecuarse tanto a la dinámica nacional, como a la internacional. En este sentido, es posible identificar dos grandes procesos determinantes para el arreglo institucional del desarrollo en México y que se expresan a través de la política social. El primero, podemos identificarlo como tradicional o posrevolucionario pues se caracterizó por obedecer a una lógica en donde lo fundamental, fue articular el mundo del trabajo con los servicios de provisión y asistencia. El segundo, surgido a partir de 1983 con base en la reordenación económica y el cambio estructural, sigue vigente y se presenta como una forma de regular, proveer y promover servicios con calidad, equidad, eficiencia y competitividad a partir de una nueva relación entre Estado, mercado y sociedad. Entre los resultados de este último proceso destaca el papel y la función adoptada por cada uno de los actores. Así, el Estado, se muestra como regulador y promotor de la equidad en términos de

6 Tomo como referencia el concepto de Douglass C. North, sobre las instituciones en las sociedades como estructura básica por medio de la cual la humanidad a lo largo de la historia ha creado orden y de paso ha logrado reducir la incertidumbre. Asimismo, me identifico con su idea de que las instituciones son el determinante subyacente del desempeño de las economías. Ver: Instituciones, cambio institucional y desempeño económico, México, Fondo de Cultura Económica, 1993. 
servicios, costos y calidad. Por su parte, el mercado, consolida las diferencias sociales al impedir el acceso de bienes y servicios a ciertos grupos de la sociedad, mientras la sociedad, advierte una polarización más profunda frente a lo cual la política social se focaliza hacia el extremo con mayores necesidades.

Tenemos pues, que las acciones y los programas sociales tienden a dirigirse hacia la población vulnerable, cuyas expectativas se han visto desvanecerse. Destacan, entre ella los jóvenes, quienes tienen frente a sí un conjunto de problemáticas sociales que impiden su realización plena como ciudadanos y como forjadores de su propio futuro.

\section{Condición juvenil en México: los ámbitos de la educación, el trabajo y la participación} política

Proyecciones socio-demográficas ${ }^{7}$ calculan una población total aproximada de 112 millones de habitantes en México para el 2015. Según datos obtenidos del Instituto Nacional de Estadística, Geografía e Informática (INEGI) la población juvenil en el 2015 será de aproximadamente 29 millones $519 \mathrm{mil}^{8}$, es decir, $27 \%$ pertenecerá al grupo etario de 14 a 29 años de edad.

Estas cifras y otras dadas a conocer por el INEGI a propósito del día mundial de la juventud en el $2011^{9}$ confirman, entre otras cosas que, pese a haberse reducido la tasa de natalidad la población mexicana sigue manteniendo una fuerte presencia juvenil. Al año 2010, el grupo de 15 a 19 años lo integran más de 13 millones de personas, lo que representa algo más de $12 \%$ de la población total del país; mientras que el más numeroso es el rango de 20 a 29 años que ocupa $18.4 \%$ del total. En total estamos hablando de un grupo poblacional que va de los 15 a 29 años que representa alrededor de $30 \%$ de la población total al último censo de 2010.

En relación con las condiciones que enfrentan estos jóvenes y que caracterizan al país, datos del último Informe de Desarrollo Humano (PNUD, 2011) ubican a México en el lugar número 57 del Índice de Desarrollo Humano, con un desarrollo medio definido por un valor de 0.770. Algunos indicadores sobre la condición socioeconómica nacional que se presentan en el Informe, señalan seis elementos que definen el periodo 2000 y 2010 :

a) $3.4 \%$ de la población vivía con 1,25 dólares diarios, mientras que el porcentaje de la población que vive por debajo de la línea de pobreza nacional es del $47.4 \%$.

b) Sólo $10 \%$ más pobre de la población, participa con 1,8\% del PIB mientras $10 \%$ más rico, con $37,9 \%$.

c) En el territorio nacional, 6.6\%. de la población mayor a los 15 años de edad, en el 2010, era

\footnotetext{
${ }^{7}$ Las proyecciones fueron realizadas por el Consejo Nacional de Población (CONAPO) para el 2005 y estuvieron basadas en el Censo de Población y Vivienda que se aplicó en el año 2000. Los resultados aparecieron publicados en el proyecto México en cifras: indicadores demográficos básicos, República Mexicana, 1990 -2050 a través del portal: http://www.conapo.gob.mx/00cifras/proy/ Proy05-50.pdf y fueron actualizados en el documento Proyecciones de la población en México 2005-2050, CONAPO, México, 2006: http://www.conapo.gob.mx/es/CONAPO/Proyecciones de la poblacion_de Mexico_2005-2050

${ }^{8}$ Los datos sobre población juvenil fueron obtenidos del último Censo de Población y Vivienda 2005. Consultar: www.inegi.gob. mx/est/contenidos/espanol/rutinas/ept.asp y fueron complementados con algunos publicados en los Resultados Preeliminares de la Encuesta Nacional de Juventud 2005: Consultar: www.imjuventud.gob.mx/temas.asp. Es importante señalar los datos presentados son aproximados toda vez se carece de resultados coincidentes entre las diversas instancias de gobierno que realizan estudios sobre población en México.

${ }^{9}$ Para ampliar esta información puede consultarse:

http://www.inegi.org.mx/inegi/contenidos/espanol/prensa/contenidos/Articulos/sociodemograficas/mexico-jovenes.pdf
} 
analfabeta.

d) El porcentaje de la población sin acceso sostenible a fuentes de agua al año 2009, fue de 9\%; mientras que en relación al saneamiento mejorado es de $2 \%$.

e) En la población infantil, $5 \%$ de los niños nacen con bajo peso y $8 \%$ de los chicos menores de 5 años de edad, mantienen un peso inferior al normal y $18 \%$ refleja una estatura inferior.

f) La tasa de desempleo en abril de 2010, representó $5.5 \%$ de la población económicamente activa

De cara a éstas condiciones y con una población que alcanzará los 112 millones de habitantes para el año 2015, sin duda atender la cuestión social es una tarea urgente para garantizar un entorno que favorezca el pleno desarrollo de las capacidades juveniles en el mediano y corto plazo.

Son diversas las tareas y múltiples los actores necesarios para hacer frente a la situación que aqueja a los jóvenes, por lo que parece sustantiva la responsabilidad gubernamental. En este sentido, el diseño de las políticas juveniles juega un papel decisivo pues, México se caracteriza por respaldarse en modelos tradicionales que por lo general, enfocan la etapa juvenil como una de transición hacia la adultez y no logran visualizar a la juventud como sujeto social del presente.

Entre otras cosas, ello profundiza el abismo entre el mundo joven y el mundo adulto, pero al mismo tiempo, esta distancia desdibuja los límites entre cada etapa y hace complejas sus relaciones. Así, algunos rasgos de la participación juvenil mexicana coinciden con aquellos referidos por la Comisión Económica para América Latina (CEPAL, 2004 y 2008) como característicos de la región Latinoamérica:

1. Descrédito y poco interés en las instituciones políticas y sus actores;

2. Descrédito al sistema democrático;

3. Mayor nivel de asociatividad juvenil en ciertas prácticas culturales tradicionales (religiosas y deportivas);

4. Creciente importancia de modalidades asociativas informales;

5. Disociación entre un alto nivel de conciencia respecto de problemas de ética social y un bajo nivel de participación;

6. Importancia de los medios de comunicación en la asociatividad juvenil;

7. Tendencia incipiente a opinar y participar en temas de interés público mediante la conexión a redes virtuales;

8. Mayor tendencia a participar en organizaciones de voluntariado que en organizaciones políticas.

9. Tendencia a la articulación horizontal, autónoma y autogestiva.

Estos aspectos y las nuevas formas de agrupación que los identifican, no sólo son anulados por las políticas tradicionales, sino que su ausencia impide la construcción de canales y mecanismos que alienten y den sentido a la participación de los jóvenes. Así, las tendencias vigentes buscan evitar que los jóvenes sean solo receptores del cambio político y social. Por ello, además de las tradicionales y siguiendo a Krauskopf (2003) actualmente, se incluyen dos modelos más para las políticas juveniles: el transicional y el avanzado. 
Las políticas transicionales, contemplan a los jóvenes como sujeto juvenil desde los problemas que amenazan el orden social o desde el déficit en las manifestaciones de su desarrollo. Las políticas avanzadas, se orientan al empoderamiento de los jóvenes como ciudadanos y actores estratégicos del desarrollo.

A partir de este marco de referencia y por su impacto en la sociedad, en este trabajo presentamos algunos avances y retrocesos que en materia juvenil caracterizan a México, en el ámbito educativo, laboral y de participación política.

\section{La esfera de la educación}

La educación ha sido por tradición la política de juventud que ha preocupado más a los gobiernos mexicanos. Particularmente, éstas se han centrado en abatir los niveles de analfabetismo y la deserción escolar. Hacia 1997, los niveles de matriculación se incrementaron hasta alcanzar el $97 \%{ }^{10}$ en el Sistema Educativo Nacional de acuerdo a datos del INEGI para ese año, manteniendo un equilibrio relativo entre hombres y mujeres. Es importante mencionar que el promedio general de escolaridad de los jóvenes en esos años, fue de 8.5 grados, tanto para hombres como para mujeres. Sin embargo, las diferencias educativas entre la población juvenil rural y urbana continuaban siendo significativas, mientras que en el ámbito rural su promedio de escolaridad era 6.5 años, en las localidades urbanas ascendía a 9.2 años. Asimismo, en el medio rural $43 \%$ de los hombres y $40 \%$ de las mujeres jóvenes tenían estudios de secundaria o más, mientras que en el medio urbano $79 \%$ de los hombres y $76 \%$ de las mujeres jóvenes tenían estudios de secundaria o más. Por otra parte datos del INEGI para el 2006 señalaron que el abandono escolar de los jóvenes entre los 15 y 24 años de edad es una situación que no se ha logrado abatir de forma tal que, sólo $32 \%$ de la población, de 15 a 24 años, continúa inscrita en el sistema educativo nacional, de los cuales $34 \%$ son varones y $30 \%$ mujeres. Según datos de la Encuesta Nacional de la Juventud $2005^{11}$ el nivel de educación básica era completado por $12 \%$ de las mujeres entre los 12 y 29 años de edad, mientras que solamente un $9 \%$ de los hombres es aquel que concluye este ciclo educativo.

Casi diez años después estas cifras se habían modificado. En su Primer Informe de Gobierno, el presidente Calderón señalaba que $98 \%$ de la población joven sabía leer y escribir, los hombres (98.2\%) y las mujeres (97.9\%) presentan una proporción similar. Por entidad federativa el comportamiento de este indicador era contrastante: en el Distrito Federal $97.6 \%$ de los jóvenes son alfabetas, mientras que en Chiapas sólo sabía leer y escribir $79.6 \%$ de la población joven.

\section{El ámbito del Empleo}

De acuerdo con la Encuesta Nacional de la Juventud 2005 la población juvenil cuya única actividad era estudiar representó $38.8 \%$ del total. Asimismo, de acuerdo con ésta Encuesta, las y los jóvenes que no estudian ni trabajan se aproxima a la cifra de 8 millones. Vale la pena mencionar,

\footnotetext{
${ }^{10}$ Es importante señalar, que al hablar de los niveles de matriculación contemplamos la estructura de medición que señala el INEGI. Pare el INEGI, la matrícula se refiere al conjunto de alumnos inscritos en un ciclo escolar en cualquiera de los niveles del Sistema Educativo Nacional, que comprende tres tipos de educación: básica, media superior y superior (preescolar, primaria, secundaria, bachillerato, licenciatura y posgrado).

${ }^{11}$ Consulta en línea:

http://www.imjuventud.gob.mx/imgs/uploads/Encuesta_Nacional de Juventud_2010 - Resultados Generales 18nov11.pdf
} 
las más afectadas por esta circunstancia son las mujeres ya que de ese total, ellas representan $75.7 \% .^{12}$

En el mismo documento y considerando a la población juvenil con trabajo, se destacó que sólo dos de cada 10 jóvenes lo hacían en un empleo relacionado con sus estudios. Por lo demás, las condiciones laborales que enfrentaban eran muy precarias, ya que sólo $29.3 \%$ de los jóvenes contaba con un contrato de trabajo y eran pocos los que vivían en una estabilidad laboral, pues la mayoría eran contratados por obra determinada o en calidad de eventuales o de confianza. Por otra parte, se reportó que la tasa de desempleo entre los jóvenes es tres veces mayor que la de los adultos y $70 \%$ de los trabajos se encuentran en el sector informal, siendo que $28 \%$ de los jóvenes que no estudian ni trabajan se encuentran en la búsqueda de oportunidades de empleo. Concretamente en el periodo 2000-2004 se remarcaron las debilidades en el mercado de trabajo y el empleo nacional creció 1.7\% por año (Ibarra, 2005).

La Encuesta Nacional de Juventud 2010 (ENJ-2010) reportó que el número de jóvenes que sólo estudian disminuyó cinco puntos porcentuales al ubicarse en 38.8\%. Asimismo, reveló que $22 \%$ no estudia, ni trabaja. La proporción de jóvenes que trabajan y aportan a la economía familiar son 8 de cada 10. De éstos $60 \%$ respondió aportar la mitad o menos de su salario y $24 \%$ todo lo que gana. Por su parte datos del INEGI en el 2010 revelan que, durante el primer trimestre de 2010, la población económicamente activa (PEA) del país entre los 15 y 29 años ascendió a 13.9 millones de personas, cifra que representa $27.8 \%$ de la fuerza de trabajo total. Dicha proporción es inferior en 4.7 puntos porcentuales a la registrada en el mismo trimestre de 2009.

Así, la desocupación entre los jóvenes entre 2006 y 2009 pasó de 904 mil a 1 millón 248 mil, lo que significó un crecimiento de 344 mil desocupados en éstos años, es decir, la tasa de desocupación durante el periodo creció de $6.2 \%$ a 8.5 por ciento. Este mismo fenómeno afectó más a las mujeres jóvenes cuya tasa de desocupación, según la ENJ-2010, representaba 9.8 \% mientras que en hombres representaba 41.1\%. Según la jornada de trabajo, 877 mil jóvenes trabajan menos de 15 horas semanales, cifra que representa $6.5 \%$ del total de jóvenes ocupados y en el extremo contrario se ubican 3.6 millones de jóvenes que laboran más de 48 horas semanales, equivalente 27.1 por ciento. En cuanto a sus ingresos, 39\% de los jóvenes percibe hasta 2 salarios mínimos, $23.5 \%$ más de 2 y hasta 3 salarios mínimos, y $20.7 \%$ obtiene más de 3 salarios mínimos por su trabajo; en cambio, $10.6 \%$ no recibe ingresos y $6.2 \%$ no declaró sus ingresos. Ello significa que los jóvenes abandonan sus estudios para incorporarse al mercado de trabajo y buscar mejores condiciones de vida para ellos y sus familias.

\section{El espacio de la participación juvenil}

En relación con esta temática, nos referiremos sobre todo a la participación política de los jóvenes. Para el 2009 y considerando la totalidad de población juvenil, datos del INEGI señalaban que $83.1 \%$ poseía credencial de elector y del mismo total, casi ocho de cada 10 habían votado alguna vez y, 67.8\% votaron en las últimas elecciones (se refiere a las del 2006).

Con respecto a la confianza que los jóvenes tienen por las instituciones, entre las Encuestas

\footnotetext{
${ }^{12}$ Si se quiere profundizar en el tema sobre mujeres, consultar: Florinda Riquer y Ana María Tepichin, Mujeres jóvenes en México. De la casa a la escuela, del trabajo a los quehaceres del hogar, consultado en:

http://www.uia.mx/campus/publicaciones/jovenes/pdf/epieck16.pdf
} 
2000 y 2005 se observaron ciertos reacomodos en sus preferencias. Mientras en la del 2000, la familia y la iglesia absorbieron los mayores porcentajes (alrededor de una tercera parte confiaba en ellas) ahora lo son, la familia en primer lugar con 9.1 de calificación sobre un máximo de 10 y la escuela, con 8.3, mientras que los sacerdotes, curas y ministros religiosos descendieron a un nivel intermedio con un puntaje de 7.7. Por otro lado, las instituciones con menores niveles de confianza continúan siendo los partidos políticos y el Congreso pero se suma la policía con una calificación de 5.9. Asimismo $44 \%$ de los jóvenes reportó desinterés en la política.

Lo anterior también se ve reflejado en la reciente Encuesta Nacional de la Juventud del 2010, donde se refleja que entre la mayor parte de los jóvenes, $63.1 \%$ opina que los partidos políticos son poco o nada necesarios para la funcionalidad democrática. Al mismo tiempo, la participación juvenil se deja ver escasa al ser sólo $11 \%$ de los jóvenes los que participan a través de alguna organización o asociación civil como elemento de incidencia complementario al voto.

Sin duda, la situación educativa y laboral para los jóvenes mexicanos es por demás desalentadora, lo cual incide entre otras cosas en su baja participación y en la búsqueda constante de espacios donde puedan ejercer sus libertades. Los mecanismos instrumentados hasta ahora para alentar su intervención en la resolución de los problemas que le aquejan son percibidos como lejanos a sus necesidades. Al respecto $99.4 \%$ de los jóvenes consideran que la democracia no promueve ni el acceso a la educación, ni el mejoramiento de los servicios educativos, esto a pesar de la apreciación mayoritaria que expresa la ENJ-2010 sobre el acuerdo que existe entre los jóvenes en referencia al ejercicio del voto (76\%) y a los datos arrojados por el INEGI, donde en el 2011 indicaban que $30.41 \%$ del padrón estaba representado por jóvenes con edad para votar.

\section{El tema de la juventud en la agenda nacional y la puesta en marcha de políticas juveniles}

México es uno de los países con mayor tradición en el trabajo relacionado con jóvenes, pues ya desde mediados del siglo XX cuenta con instituciones para tal fin. En 1942 surge, dependiente de la Secretaría de Educación Pública, la Oficina de Atención Juvenil. Posteriormente, desde 1950 hasta fines de los 60, la responsabilidad de las políticas juveniles se le confiere al Instituto Nacional de la Juventud Mexicana, el cual, mediante diversos programas de capacitación buscó atender las necesidades de los jóvenes, suponiendo que su necesidad central era la de integrarse a la sociedad asumiendo las responsabilidades de la vida adulta. En 1976 se produce un cambio cualitativo cuando se constituye el Consejo Nacional de Recursos para la Juventud (CREA) que empezaría a concebir las políticas de juventud como competencia no sólo del ejecutivo federal, sino como tarea de la sociedad y de los tres órdenes de gobierno ${ }^{13}$. Entre 1988 y 1994, las políticas federales de juventud en México sufren un recorte importante, ya que fueron subsumidas en la Comisión Nacional del Deporte (CONADE). Aunque entre 1993 y 1995, se desarrolla el Programa de Jóvenes en Solidaridad (PJS) no sería sino hasta 1996, cuando las políticas de juventud tendrían un nuevo aliento a través de la creación de Causa Joven, cuyos méritos fueron generar en los gobiernos estatales la necesidad de crear estructuras de gobierno propias y especializadas en el desarrollo de programas de juventud.

Algunos autores como Domínguez y Morales (2005) consideran, las principales características

\footnotetext{
${ }^{13}$ Con base en el documento Jóvenes e instituciones en México 1994-2000, publicado por el Instituto Mexicano de la juventud en el año 2000, existieron un CREA en cada estado de la República; 1122 municipales; y, 55 regionales.
} 
de las políticas juveniles en México de 1942 a 1998 fueron:

a) Su centralismo, basado en su origen federal

b) Su enfoque universal, definido a partir de la pretensión por establecer políticas de carácter nacional,

c) Su constante dependencia de las circunstancias políticas, nacionales o estatales, dado las instituciones de juventud fueron creadas y liquidadas por decretos presidenciales e impulsadas en los estados por los ejecutivos estatales. Ello, entre otras cosas, las vulnera frente a la renovación de los gobiernos.

d) Su enfoque sectorial en el tratamiento a los jóvenes, bajo una lógica profundamente paternalista y tutelar.

Por lo que a la historia reciente de las políticas juveniles se refiere, podemos decir que inicia cuando en 1999 se aprueba por Decreto Presidencial la creación del Instituto Mexicano de la Juventud (IMJUVE). El Instituto surge como organismo público descentralizado, con personalidad jurídica, patrimonio propio, cuyo objetivo es definir e instrumentar una política nacional de juventud que permita incorporar plenamente a los jóvenes, entre los rangos de edad de 12 a 29 años, al desarrollo del país. A partir de la creación del IMJ, surgieron en algunos estados organismos similares y especializados en las políticas de juventud estatal. Actualmente existen 22 Institutos Estatales.

Para el logro de su objetivo, se dotó al Instituto de funciones para articular planes, programas y proyectos que involucraran esfuerzos institucionales, así como para generar conocimiento y difundir información para la juventud. Entre las acciones más destacadas se encuentra la creación del Centro de Estudios e Investigaciones sobre la Juventud Mexicana (CIEJUV) quien además, tuvo a su cargo la realización de la Encuesta Nacional de la Juventud Mexicana (2000) y coadyuvó en la formulación del Programa Nacional de Juventud (2002-2006) desde una óptica que asumía que los jóvenes son sujetos de derechos y actores estratégicos del desarrollo.

Esta idea de promover la construcción de ciudadanía en los jóvenes, tiene como antecedente los estudios elaborados por la Organización Internacional de la Juventud (OIJ) en el Marco de Referencia del Programa Regional de Acciones para el Desarrollo de la Juventud en América Latina (PRADJAL) elaborado para el periodo 1995-2000 (Pérez Islas, 1995). En dicho documento, se propusieron dos caminos para desarrollar las políticas de juventud en la región: el primero puede denominarse específico y buscó articular una serie de programas con otras instituciones $u$ organizaciones que confluyeran en su desarrollo, tal es el caso de modelo mexicano emprendido antes de 1985. La segunda opción, que llamaremos integrada, más bien fue y es desarrollada en países como Cuba, donde sin existir una centralidad institucional, la atención a jóvenes se lleva a cabo en diversas instancias de acuerdo a su campo de trabajo, para lo cual se necesita una gran conciencia en todos los niveles con el fin de no olvidar al sector juvenil.

Ya puesto en marcha el Plan Nacional de la Juventud 2002-2006, en México, la OIJ junto con la CEPAL (2004) dan a conocer su Primer Informe Iberoamericano sobre la Juventud ${ }^{14}$ en donde ofrecen un diagnóstico cuantitativo y cualitativo sobre las contrariedades que afectan a la población

${ }^{14}$ Este estudio contempla 17 países. Quince de ellos en el continente americano: Argentina, Bolivia, Chile, Colombia, Costa Rica, Cuba, Ecuador, Guatemala, México, Nicaragua, El Salvador, Panamá, Perú, República Dominicana y Uruguay. Dos más, en el continente europeo: España y Portugal. La información puede consultarse en: Comisión Económica para América Latina y el Caribe (CEPAL), Naciones Unidas, Organización Iberoamericana de Juventud (OIJ), Primer Informe Iberoamericano sobre la Juventud, La Juventud en Iberoamérica: tendencias y urgencias, Santiago de Chile, octubre de 2004. 
juvenil en Latinoamérica. Dichas desilusiones o desencantos, se agruparon en siete paradojas:

1. Más acceso a educación y menos acceso a empleo

2. Más aptitud para el cambio productivo, pero más exclusión de este.

3. Más expectativas de autonomía y menos opciones para materializarla

4. Mayor acceso a la salud y menor reconocimiento de morbimortalidad ${ }^{15}$ específica

5. Más acceso a información y menos acceso al poder

6. Mayor adaptabilidad y movilidad, pero mayores inconvenientes por trayectorias migratorias inciertas

7. Mayor cohesión hacia adentro, pero con mayor impermeabilidad hacia fuera

Con ello, el énfasis en el diseño y formulación de políticas juveniles se incorporaba con gran determinación en la agenda gubernamental. Además de este esfuerzo multinacional, por resaltar la problemática que actualmente viven los jóvenes, durante la XII Conferencia Iberoamericana de Ministros de Juventud que se llevó a cabo en el mes de noviembre de 2004 en la ciudad de Guadalajara, en México, se promovió llevar a cabo una Convención Iberoamericana de Derechos de los Jóvenes. Esta tuvo como sede para su firma, la ciudad de Badajoz, España ${ }^{16} y$ dio como resultado un tratado internacional en dicha materia. Así, durante el año 2005 tuvieron lugar tres hechos destacados para la construcción de políticas de juventud en lberoamérica. Por una parte, la celebración del XX Aniversario del Año Internacional de la Juventud. (AIJ) por Declaración de las Naciones Unidas. Por otra, la conmemoración de los diez años del Programa Regional de Acciones para el Desarrollo de la Juventud en América Latina (PRADJAL). Por último, la celebración de la Primera Convención Iberoamericana de Derechos de los Jóvenes.

Un año más tarde, en la Cumbre lberoamericana de Ministros de Juventud celebrada en Cuba, se ratificó la Convención Iberoamericana de Derechos de la Juventud; se establecieron los plazos para la elaboración del Plan de Cooperación e Integración de la Juventud en Iberoamérica; se compartió un modelo unificado y racionalizado de estadísticas e indicadores en materia de juventud; y se impulsó la creación del Programa de Fortalecimiento Institucional en Políticas Públicas de Juventud Rural y la puesta en marcha de una Comisión Iberoamericana de Juventud para el Desarrollo Sustentable del Medio Ambiente en el ámbito de la OIJ, presidida por México.

Durante la última Conferencia Iberoamericana de Ministros de Juventud, que tuvo lugar en el 2010 en Santo Domingo, República Dominicana, se acordó la creación de una Agencia especializada para la Juventud cuyo objetivo será coordinar la cooperación internacional dirigida a la protección de los derechos de los y las jóvenes y al desarrollo de políticas públicas integrales. También, fueron ratificados los acuerdos de la Declaración de Guanajuato en la Conferencia Mundial de Juventud de 2010 que tuvo lugar el 27 de agosto y donde se estableció la celebración del Año Internacional de la Juventud con el lema "Diálogo y Entendimiento Mutuo" del 12 de agosto de 2010 al 11 de agosto de 2011.

En la última década, la participación de México en los organismos internacionales ha sido

\footnotetext{
${ }^{15}$ En el Diccionario de la Lengua Española, editado por la Real Academia de la Lengua en su Vigésima Segunda edición, 2001, la palabra Morbilidad (del inglés, morbility) se refiere a la proporción de personas que enferman en un sitio y tiempo determinado. Por su parte, Mortalidad (del latín mortalitas) describe la tasa de muertes producidas en una población durante un tiempo dado, en general o por una causa determinada. Así, morbimortalidad, es la proporción de personas que enferman y mueren en un tiempo determinado.

${ }^{16}$ Para consultar los resultados de la Convención:

http://www.xvicumbre.org.uy/pdf/cumbre2005/acta convencion_jovenes.pdf
} 
constante, sin embargo, el impacto de su actividad en el ámbito nacional no ha sido destacado.

Con este marco de referencia, el Programa Nacional de Juventud 2008-2012 (PNJ) elaborado durante la gestión gubernamental del actual Presidente de la República, el Lic. Calderón Hinojosa, propone líneas generales que poco abonan en la modificación de las estructuras de antaño. En su conjunto, el documento busca generar un enfoque generacional en todas y cada una de las acciones y programas del gobierno en sus tres ámbitos. Para su puesta en marcha, cuenta con cinco ejes operativos:

1. Conocimiento: Que incluye sustentar el diseño y ejecución de las políticas de juventud en el saber generado por la investigación permanente sobre las realidades juveniles, con el fin de dar seguimiento a sus necesidades y demandas, para asegurar la pertinencia de las acciones que llevan a cabo las instituciones y organizaciones involucradas en dicha política.

2. Coordinación: Como mecanismo para conjuntar y articular las acciones, experiencias y recursos en materia de juventud de los diversos actores sociales y niveles de gobierno, con el propósito de obtener el mayor impacto en los jóvenes, a través de un mapa de recursos institucionales y sociales

3. Comunicación: Que integra las acciones para informar con transparencia a la sociedad sobre las acciones desarrolladas en materia de juventud, y concretar espacios para la expresión e interacción con la población juvenil y entre ellos mismos, con el fin de establecer canales permanentes de diálogo entre el gobierno, la sociedad y los jóvenes.

4. Legislación: Cuando así lo requiera la temática, proponer al Poder Legislativo trabajar de manera conjunta en la generación de un marco legal adecuado para instrumentar leyes y demás disposiciones de carácter general, que promuevan el desarrollo integral de los jóvenes.

5. Evaluación: Integrar las acciones de seguimiento derivadas del PNJ, con el propósito de medir su eficiencia e impacto en la población juvenil para su permanente actualización a través de la composición de indicadores por objetivos.

De igual forma el PNJ está conformado por seis objetivos generales:

1) Ciudadanía y participación social. Promover la participación de las y los jóvenes, por medio de la protección y el reconocimiento de su ciudadanía.

2) Acceso efectivo a la justicia. Impulsar el ejercicio efectivo de los derechos de las y los jóvenes reconocidos en la legislación nacional, a través de su acceso al aparato de procuración e impartición de justicia.

3) Acceso y permanencia en la educación. Garantizar el derecho de las y los jóvenes a una educación pertinente y de calidad, mediante su acceso y aprovechamiento en condiciones equitativas.

4) Trabajo decente. Salvaguardar el derecho de las y los jóvenes al trabajo, a través de su integración laboral en condiciones de trabajo decente.

5) Fomento de la salud de las y los jóvenes. Promover el acceso equitativo a los servicios de salud pública y el fomento de estilos saludables de vida.

6) Vivienda adecuada. Propiciar el acceso de las y los jóvenes a una vivienda adecuada, por 
medio de la promoción de sus necesidades en las políticas y programas de vivienda.

Sobre estos ejes generales, la búsqueda y construcción de un entramado institucional que de cabida a la resolución de los problemas que atañen a los jóvenes y a sus expectativas, se desdibujan. Entre las principales políticas de juventud que han sido promovidas por el actual gobierno en los ámbitos de la educación y el empleo, destacan las siguientes:

\section{Políticas de juventud para la educación}

Un antecedente relevante en el ámbito de la educación, fueron los resultados de las pruebas nacionales de lectura y matemáticas aplicadas a finales del ciclo escolar 2002-2003 a los alumnos de secundaria. En ellas, solo 30 de cada 100 alumnos demostraron tener mayor dominio de los contenidos de las pruebas de lectura y apenas 19 de cada 100 dominaron las pruebas de matemáticas. Asimismo y de acuerdo con los resultados de la aplicación de las pruebas en el 2003 del Programa para la Evaluación Internacional de los Estudiantes (PISA) promovido por la Organización para la Cooperación y el Desarrollo Económicos (OCDE) a estudiantes del tercer grado de secundaria, la media nacional en las escalas de lectura, matemáticas y ciencias fue significativamente menor a la obtenida por el conjunto de países miembros. Las cifras reflejaron que de un promedio en la OCDE de 494, 500 y 500 puntos en lectura, matemáticas y ciencias, respectivamente, la media nacional en puntos fue de 392 para lectura, 378 para matemáticas y 397 para ciencias (SEP, 2003).

Para contrarrestar esta situación, se promovió la Reforma Integral de la Educación Media Superior (RIES) a través de la Secretaría de Educación Pública (SEP, 2005). Los objetivos establecidos por la reforma fueron cuatro: la construcción de un marco curricular común; la definición y reconocimiento de las porciones de la oferta de la Educación Media Superior; la profesionalización de los servicios educativos; y, la Certificación Nacional Complementaria. Para el cumplimiento de las metas, se impulsaron dos procesos participativos:

a) El primero consistió en una Consulta Nacional para promover la reflexión y debate entre maestros, alumnos, directivos, personal de apoyo y otros actores sociales y académicos. Dicha consulta, se llevó a cabo del 22 de agosto al 4 de noviembre del 2005.

b) El segundo, que se llevó a cabo del 22 de agosto del 2005 al 7 de julio del 2006, contempló en el ciclo escolar 2005-2006 la puesta en marcha de una reforma curricular en 150 escuelas muestra.

La RIES, impulsada aún durante el periodo gubernamental del Presidente Vicente Fox, se encuentra en proceso y se estima concluya durante el año 2012, al término de la gestión del Presidente Calderón Hinojosa.

La política educativa actual está enmarcada en un documento básico que es el Programa Sectorial de Educación 2007-2012, el cual se desprende del Programa Nacional de Desarrollo. Dicho programa sectorial descansa sobre tres subprogramas: educación básica, educación media superior y superior. Entre sus objetivos generales se señala avanzar hacia la equidad de la educación; proporcionar una educación de calidad adecuada a las necesidades de los mexicanos e impulsar el federalismo educativo, la gestión institucional y la participación social. 
Otra política relevante en este periodo, se relaciona con el establecimiento en el año 2010 de la Alianza por la Calidad de la Educación, suscrita por el gobierno federal a través de la Secretaría de Educación Pública (SEP) con el Sindicato Nacional de Trabajadores de la Educación (SNTE). La Alianza tiene como objetivo, impulsar una transformación por la calidad de la educación del Sistema Educativo Nacional. Los avances y resultados pueden consultarse a través de los informes mensuales que desde el mes de junio del 2010 y hasta la fecha, se publican.

\section{Políticas de juventud para la generación de empleo y oportunidades}

Desde principios de los años ochenta las autoridades laborales y educativas mexicanas establecieron algunos principios rectores de las políticas y programas destinados a apoyar la formación y el empleo de la población joven que se encuentra próxima a incorporarse al mercado de trabajo o que no ha logrado insertarse de manera estable en una actividad laboral remunerada. Existen por lo menos cuatro grandes ejes rectores sobre los cuales se han sustentado estas políticas públicas:

1. La ampliación de cobertura del sistema institucional de educación y formación para el trabajo a todos los grupos de población, en particular a aquellos jóvenes que provienen de familias de bajos ingresos (pobres urbanos; pequeños productores rurales; campesinos migrantes; jóvenes con discapacidades físicas o intelectuales)

2. La consolidación en todos los estados de la República, del sistema de oficinas de vinculación que integran el Servicio Nacional de Empleo para dotarlas de una base informativa ágil y moderna que sirva como fuente confiable de consulta para el buscador de una opción de trabajo y para las empresas que demandan personal. Asimismo, la creación de las Bolsas de colocación juvenil.

3. El fomento del autoempleo y el apoyo a las micro y pequeñas unidades productivas como alternativa ocupacional frente a la insuficiente generación de puestos de trabajo que se registra en los establecimientos grandes y medianos, habitualmente identificados como el sector formal de la economía.

4. La Adaptación de la Ley Federal del Trabajo incluyendo un capítulo destinado a promover el empleo juvenil y promover política de estímulos fiscales para las empresas que contratan jóvenes

Este tipo de políticas se basaron en la idea de que la calificación de la fuerza de trabajo garantiza el ingreso al mercado laboral, sin embargo, este presupuesto teórico tuvo relativo éxito en sociedades industrializadas con altas tasas de crecimiento en las tres décadas posteriores a la segunda post-guerra, pero en países como México con altos niveles de subempleo y desocupación abierta, marcado desarrollo de las llamadas actividades informales, demuestra serias deficiencias. Asimismo, habría que señalar que a la continua precarización del mundo del trabajo se agregan, actualmente, otros factores tales como la migración. Con base en la Encuesta de la Comunidad Americana (ACS) para el 2012, el INEGI (2009) dio a conocer que del año 2000 al 2007, el volumen de jóvenes entre 15 y 29 años de edad nacidos en México y que residen en Estados Unidos de 
Norteamérica se ha mantenido cercano a los 3 millones, no obstante que el volumen total de los nacidos en México paso de 9 a casi 12 millones de personas en el mismo lapso.

\section{Hacia la construcción de nuevas políticas juveniles}

Pese a los esfuerzos emprendidos el contexto juvenil en México resulta poco alentador. Por una parte, es posible identificar una etapa transicional en la elaboración de políticas para los jóvenes en donde predomina la baja institucionalización. Es decir, la tarea para actualizar el andamiaje institucional en éste ámbito se limitó a la esfera normativa y no ha logrado consolidar instituciones sólidas que promuevan políticas avanzadas e incorporen realmente a los jóvenes. Al respecto, es posible advertir que en el plano gubernamental la mayoría de las políticas juveniles se basan aún en un modelo tradicional donde las posibilidades de los jóvenes para convertirse en generadores de cambio y forjadores de su propio futuro son escasas, relegándolos hacia un lugar ambiguo entre receptores de políticas y protagonistas del cambio.

El reto consiste en diseñar políticas que logren transitar entre los modelos tradicionales y los avanzados para sembrar y construir ciudadanía juvenil, para promover la participación de los jóvenes y para convertirlos en sujetos y actores de derecho. Se trata de cimentar una nueva relación entre el gobierno y la sociedad, donde los jóvenes puedan contribuir a delinear sus propias políticas.

Por otra parte, desde su creación, la intervención del Instituto Mexicano de la Juventud es limitada, desarticulada y con una escasa cobertura de los programas. Si bien es cierto, su constitución permitió superar una etapa importante en la formulación de políticas para la juventud en México, dicha institución debe contar con mayor liderazgo y fortaleza para influir en el conjunto de las instituciones públicas, federales, estatales y municipales. Si bien, el Programa Nacional de Juventud 2002-2006 incorporó propuestas y conceptos actualizados conforme al marco internacional, éstos no pudieron traducirse en programas y líneas de acción específicas que impactaran el ámbito juvenil.

Con el Plan Nacional de Juventud actual (PNJ2007-2012) se aprecia una política juvenil básicamente igual a la de su antecesor. En él, prevalece una lógica instrumental que focaliza los recursos hacia el sector servicios. Vale la pena mencionar también, que las diversas iniciativas legislativas para aprobar una Ley General de la Juventud en México, no han progresado.

Es innegable, la condición juvenil en México tiene "focos rojos" muy definidos que por su magnitud e impacto en la sociedad requieren de toda nuestra atención, pero de cara a este escenario existen también posibilidades deseables y posibles para revertirla. Ello supone, la modificación de ciertas estructuras de antaño, la puesta en marcha de nuevas ideas y echar a volar como dirían algunos la imaginación sociológica (C. Wrigth Mills, 1985).

Hablamos de la juventud como la esperanza del futuro. Sin embargo, la sociedad en general parece no estar consciente de la condición y situación que caracteriza a este grupo etario en la actualidad y de la necesidad no sólo de generar espacios para que sean ellos mismos quienes discutan las opciones que, como jóvenes, requieren sean proporcionadas, sino de aceptar los espacios creados por ellos mismos. Las estructuras sociales reproducen un modelo antaño de exigencia que no corresponde a las necesidades y demandas de esta población. No podemos cerrar los ojos frente al hecho de que los jóvenes de hoy viven y se enfrentan, cotidianamente, a un mundo desolador donde las oportunidades para educarse, para ingresar al mundo del trabajo y para convertirse en 
ciudadanos plenos, son cada día más limitadas. Los jóvenes deben asumir que siendo jóvenes ya deben gozar de la plena ciudadanía para construir su propia política de juventud, de acuerdo a como ellos perciben su realidad y sus expectativas. De prevalecer las paradojas actuales, los jóvenes estarán condenados a la falta de educación, el desempleo, la exclusión social, la desmovilización, la incertidumbre y la limitación de oportunidades para la toma de decisiones sobre su propia vida.

En este sentido, es importante impulsar algunas acciones al menos en seis vertientes:

I. Diagnósticos regionales y por país. Pese a los logros de algunos organismos internacionales como la CEPAL, la OIJ y la OIT para destacar la importancia de esta temática en la agenda gubernamental, resulta imperativo redoblar esfuerzos en los países. Se trata no sólo de realizar diagnósticos regionales sobre la juventud, sino también sobre cada nación a fin de identificar sus necesidades. Así, la cooperación internacional y la puesta en marcha de políticas nacionales, con programas y proyectos específicos podrán revertir las tendencias actuales y modificar, tal vez en el mediano plazo, los anhelos de la sociedad.

II. Transitar entre políticas tradicionales y avanzadas. Es importante promover un entramado institucional actualizado para enfrentar las paradojas que definen a los jóvenes de hoy. Las tendencias contemporáneas resaltan la necesidad de transitar entre los modelos tradicionales y los avanzados en el diseño de políticas sociales y en particular aquellas que se dirigen hacia la instrumentación de políticas juveniles, a fin de promover y construir ciudadanía juvenil. México, no es la excepción.

III. Impulsar la institucionalización. Es necesario consolidar el marco normativo con instituciones sólidas que den cabida a las propuestas emanadas del mismo. Actualmente, existe un vacío entre instituciones formales y la posibilidad real de echar a andar programas y líneas de acción que promuevan nuevas relaciones y vínculos entre Estado y los jóvenes.

IV. Promover la participación juvenil. Ambos, sociedad y Estado deben coordinar acciones complementarias que provean los mínimos indispensables para la vida estable, digna y duradera de la población en general. Asimismo, de forma articulada deben establecer mecanismos que permitan planear e instrumentar políticas públicas que fortalezcan la participación de los jóvenes en la sociedad, y al mismo tiempo consoliden una sociedad organizada que participe activamente en los asuntos públicos.

V. Bono demográfico. Las políticas "de los jóvenes y para los jóvenes" tendrán como marco de referencia una serie de factores económicos, políticos y sociales que determinan la condición juvenil en la región. Asimismo, podrán aprovechar el bono demográfico y las trayectorias poblacionales que por unos años, permitirán canalizar esfuerzos para revertir esta situación. Es urgente lograr prevenir los efectos que de no hacerlo, abatirían las condiciones de vida de la mayoría de la población en las próximas décadas. Ello, entre otras cosas implica, una gestión efectiva de las políticas públicas de juventud por parte de las instituciones del Estado mexicano.

VI. Evitar estigmas hacia la juventud. En efecto, es común la idea prejuiciada o estigmatizada sobre los jóvenes donde se les define como disruptores del orden social, mientras ellos se vuelcan sobre sus mundos de vida y se aíslan, cada vez más, del marco formal o institucional. 
Sin duda, la incorporación de la juventud como uno de los sectores poblacionales que requieren políticas públicas adecuadas para resolver sus diversas problemáticas ha ido ganando terreno durante los últimos años. En cada latitud, las naciones buscan establecer las bases para enfrentar su propio contexto. El caso mexicano es uno de ellos.

\section{Referencias Bibliográficas}

Diamond, L. y Morlino, L., (2005). Assesing the Quality of Democracy.USA. John Hopkins University Press.

Faundez, A. y Valverde, K. (1998) México: el difícil camino a la modernización de las políticas sociales. En, Faundez, A. (editora) Modernización de las políticas sociales en América Latina, Chile : FLACSO.

Ibarra, D. (2005) Pobreza y políticas Públicas. En, Revista Educación 2001, 125: 20-22.

Ortega Riquelme, J. M. (2006). Acuerdos tripartitas y governanza económica en México de fin de siglo. En, Foro Internacional 184, XLVI: 221-262.

Instituto Mexicano de la juventud (IMJ) (2000). Jóvenes e instituciones en México 1994-2000. México. Instituto Mexicano de la Juventud.

Krauskopf, D. (2003) La construcción de políticas de juventud en Centroamérica. En Dávila, O. (editor) En, Políticas públicas de juventud en América Latina: políticas nacionales. Santiago. CIDPA Editores.

Pérez Islas, J. A. (1995). Políticas de juventud en América Latina: evaluación y diseño. Análisis comparativo de las políticas de juventud. México. Instituto Mexicano de la Juventud.

Pérez Islas, J. A. y Maldonado, E. P. (1996). Jóvenes: una evaluación del conocimiento. La investigación sobre juventud en México 1986-1996. México, Causa Joven, Centro de Investigación y Estudios sobre juventud, 2 tomos.

Riquer, F. y Tepichin, A. M. (2001) Mujeres jóvenes en México. De la casa a la escuela, del trabajo a los quehaceres del hogar. En, Pieck, E. (coord). Los jóvenes y el trabajo. La educación frente a la exclusión social. Universidad Iberoamericana, México.

Rodríguez, E. (2004). Actores estratégicos para el desarrollo. Políticas de juventud para el Siglo XXI. México. Instituto Mexicano de la Juventud.

Valverde, K. (2007) Las políticas sociales frente a las tensiones y paradojas que enfrentan los jóvenes de hoy en América Latina. En, Juan Antonio Flores Vera (compilador) Estado, Metrópoli y políticas 
sociales. México. Plaza y Valdés Editores.

Valverde Viesca, K. y Palma, E. (2006) Ley General de Desarrollo Social: un nuevo papel para las instituciones sociales en México. En, Estudios Políticos 8, Octava Época: 9-41.

Valverde Viesca, K. (2007) Políticas de juventud para el avance educativo y la generación de empleo: las paradojas del caso mexicano. En, Medio ambiente y urbanización 66: 77-90.

Wrigth Mills, C. (1985) La imaginación sociológica. México. Fondo de Cultura Económica.

\section{Documentos}

Diamond, L. y Morlino, L. (2004) The Quality of Democracy. Center on Democracy, Development, and The Rule of Law (CDDRL) Stanford Institute on International Studies, Working Paper No. 20, 21 de septiembre.

Centro Latinoamericano y Caribeño de Demografía (CELADE) (2002) World Population Prospects: the 2002 revision. División de Población de la CEPAL, Estimaciones y Proyecciones de Población Vigente. Washington. Naciones Unidas.

Centro Latinoamericano y Caribeño de Demografía (CELADE) (2005) División de Población de la CEPAL, Serie "Transición Demográfica". En, Temas de Población y desarrollo, número 1.

Comisión Económica para América Latina y el Caribe (CEPAL) (2001) Anuario Estadístico de América Latina y el Caribe 2000. Publicado en marzo.

Comisión Económica para América Latina y el Caribe (CEPAL) (2003) y Organización Iberoamericana de Juventud (OIJ) Juventud e inclusión social en Iberoamérica, LC/R.2108/E. Noviembre.

Comisión Económica para América Latina y el Caribe (CEPAL) (2004), Naciones Unidas, Organización Iberoamericana de Juventud (OIJ) Primer Informe Iberoamericano sobre la Juventud, La juventud en Iberoamérica: tendencias y urgencias. Santiago de Chile. Octubre.

Fondo de Población de las Naciones Unidas (2005) Estado de la Población Mundial. Instituto Interamericano para el Desarrollo Social (INDES) (2005). curso en línea Condición Juvenil en América Latina. Washington, D.C. Septiembre.

Machinea, José Luis y Hopenhayn, Martin (2005), La esquiva equidad en el desarrollolatinoamericano. Serie de Informes y estudios especiales No. 14, CEPAL - Naciones Unidas. Santiago de Chile. Noviembre.

PNUD (2005), Informe de Desarrollo Humano del Programa de Naciones Unidas para el Desarrollo. 
Real Academia de la Lengua (2001) Diccionario de la Lengua Española, Vigésima Segunda edición.

Secretaría de Educación Pública (SEP) (2002), Instituto Mexicano de la Juventud (IMJ), Encuesta Nacional de Juventud 2000, Centro de Investigación y Estudios sobre, México, agosto, p. 39 y 40. Secretaría de Educación Pública (SEP) (2006) Centro de Investigación y Estudios sobre Juventud del Instituto Mexicano de la Juventud, Encuesta Nacional de Juventud 2005 (Resultados Preeliminares) Instituto Mexicano de la Juventud, México, mes de mayo.

Secretaría de Educación Pública (SEP) (2000) Programa Nacional de Educación 2000-2006, México.

Secretaría de Educación Pública (SEP) (2003) Perfil de egreso, en Consulta RIES. Consulta Nacional sobre la Reforma Integral de la Educación Secundaria. México.

Secretaría de Educación Pública (2005) "Consulta Nacional y primera fase de implementación de la Reforma Integral de la Educación Secundaria", en Revista Educare. Revista para los maestros de México. México, Año 1, num. 2, Pág. 36.

\section{Referencias electrónicas}

Consejo Nacional de Población: www.conapo.gob.mx

Declaración de Guanajuato del 27 de agosto de 2010: http://www.csa-csi.org/files/declaracion guanajuato esp.pdf

La Declaración final de la XIII Conferencia Iberoamericana de Ministros de Juventud, celebrada el 19 y 20 de octubre de 2006 :

http://www.injuve. mtas.es/injuve/contenidos.downloadatt.action?id=76448471

Krauskopf, D. (2004) La construcción de políticas de juventud en América Latina. Consultado en: http://www.gobernabilidad.cl/modules.php?name=News\&file=article\&sid=494

Domínguez, M. y Morales, H. (2005) Políticas locales de juventud en México. Inicia, Iniciativas para la Identidad y la Inclusión, Asociación Civil, México. Consultado en el Portal de Juventud para América Latina y el Caribe: http://www.joveneslac.org .

Informe de Desarrollo Humano 2005: http://www.http://undp.org Informe de Desarrollo Humano del PNUD, 2011:

http://www.undp.org/content/dam/undp/library/corporate/HDR/2011\%20Global\%20HDR/Spanish/ HDR 2011 ES Complete.pdf

Instituto Mexicano de la Juventud http://www.imjuventud.gob.mx/index.php 
Organización Internacional de la Juventud (OIJ):

http://www.oij.org/es ES/conferencias

Presidencia de la República (2007) Primer Informe de Gobierno del Lic. Felipe Calderón Hinojosa. Anexo Estadístico: 408:

http://primer.informe.gob.mx/pdf excel/P408-424.pdf 